\title{
CARACTERIZACIÓN DE LA DENSIDAD EN LAS ACCIONES COMPETITIVAS DE LOS TENISTAS DURANTE LOS PARTIDOS DE COPA DAVIS 2017 COLOMBIA VERSUS CROACIA DIA 1
}

\author{
CHARACTERIZATION OF THE DENSITY IN THE COMPETITIVE ACTIONS OF THE \\ TENNISTS DURING THE PARTIDOS OF CUP DAVIS COLOMBIA VERSUS CROATIA DAY 1
}

\author{
CARACTERIZAÇÃO DA DENSIDADE COMPETITIVA DO \\ DAVIS CUP 2017 COLÔMBIA-CROÁCIA DIA 1
}

\author{
Diego Andrés Rada ${ }^{1}$ \\ Hernán Andrés Arévalo ${ }^{2}$ \\ Diana Carolina Bustos ${ }^{3}$ \\ Alexandra González ${ }^{4}$ \\ Diego Alonso Sánchez ${ }^{5}$ \\ Jorge Enrique Buitrago ${ }^{6}$
}

Resumen

El objetivo de este artículo de investigación fue caracterizar la densidad competitiva de dos partidos de tenis
modalidad sencillos de la fase eliminatoria de la Copa Davis 2017, disputados entre el equipo de Colombia
(conformado por Santiago Giraldo y Alejandro González) y Croacia (Marin Čilić y Franko Škugor). Se utilizó
una rejilla de recolección de datos con el fin de identificar la cantidad de puntos jugados, el marcador, el tipo
de punto y la duración de cada uno de ellos para describir los tiempos totales y reales de los games y los sets;
además, establecer cuál fue el tipo de punto predominante en cada encuentro y el que menos lo fue teniendo
en cuenta cuales fueron los puntos, games y sets más largos y cortos en cada partido. Finalmente, se pudo con-
cluir que el resultado de los encuentros fue dado por las condiciones físicas de orden explosivo de los tenistas
y por su eficiencia en la precisión de ubicación de la bola y en la rápida definición de los puntos, de manera
que fueron predominantes los puntos cortos.

Palabras clave: caracterización de la competición; copa Davis; tenis; densidad.

\section{Abstract}

The objective of this article was to characterize the competitive density of two simple modal tennis matches of the qualifying round of the 2017 Davis Cup played between the Colombia team (Alejandro González y Santiago Giraldo) and Croatia team (Marin Čilić and Franko Škugor). A data collection grid was used to identify the number of points played, the marker, the type of point and the duration of each of them to describe the total and actual times of the games and sets; besides of establishing which was the type of predominant point

1 Maestrante ciencias del deporte Universidad de Ciencias Aplicadas y Ambientales UDCA, Colombia, drada@udca.edu.co. https://orcid. org/0000-0001-6536-222X

2 Maestrante en ciencias del deporte UDCA, Colombia, herarevalo@udca.edu.co. https://orcid.org/0000-0003-2534-9965

3 Maestrante en ciencias del deporte UDCA, Colombia, dibustos@udca.edu.co, Docente Universidad Javeriana. https://orcid. org/0000-0002-5632-662X

4 Maestrante en ciencias del deporte UDCA, Colombia, alexacone_40@hotmail.com. https://orcid.org/0000-0002-2274-6124

5 Magíster en metodología del entrenamiento deportivo. Instituto Superior de Cultura Física "Manuel Fajardo" de Cuba. disanchez@udca. edu.co Docente Universidad de Ciencias Aplicadas y Ambientales. https://orcid.org/0000-0003-4110-1572

6 Doctor en Ciencias de la Cultura Física (Universidad de Ciencias de la Cultura Física y el Deporte "Manuel Fajardo" de Cuba), jorge.buitrago@udca.edu.com. Docente Universidad de Ciencias Aplicadas y Ambientales. https://orcid.org/0000-0003-4490-7774 
in each match and the one that was less predominant considering all the points, games and longer and shorter sets in each match. It could be concluded that the result of the matches was given by the physical conditions of the explosive order of the tennis players and by their efficiency in the precision of the location of the ball and in the quick definition of the points, so that they were predominantly the short points.

Key words: characterization of the competition; Davis Cup; tennis; density.

\section{Resumo}

O objetivo deste artigo de pesquisa foi caracterizar a densidade competitiva de duas partidas de tênis de modalidade simples da fase eliminatória da Copa Davis de 2017, disputadas entre a equipe da Colômbia (composta por Santiago Giraldo e Alejandro González) e a Croácia (Marin Čilić e Franko Škugor). Uma grade de coleta de dados foi usada para identificar a identificação do número de pontos jogados, o marcador, o tipo de ponto e a duração de cada um deles para descrever o tempo total e real dos jogos e sets; Além disso, estabeleça qual foi o tipo de ponto predominante em cada partida e o menos frequente, considerando os pontos mais longos e mais curtos, jogos e sets de cada partida. 0 resultado das partidas foi dado pelas condições físicas explosivas dos tenistas e por sua eficiência na precisão da localização da bola e na rápida definição dos pontos, com predominância de pontos curtos.

Palavras-chave: caracterização da competição; copa Davis; tênis; densidade.

Fecha de recepción: - de enero de -

Fecha de evaluación: - de junio de -

Para citar este artículo:

???. Lúdica Pedagógica, 29, 105-114. https://doi.org/10.17227/ludica.num29-??? 


\section{INTRODUCCIÓN}

La necesidad de identificar las principales características del accionar competitivo ha sido argumentada desde varios autores como Zatsiorsky, V. (1989), quien expresa que estas cualidades permiten determinar las tendencias de trabajo de entrenamiento y la selección de los deportistas. Desde el voleibol, Bosco, Forteza, Martínez y Herrera, citados por Buitrago, J. (2016) refieren que el análisis por separado del tipo de movimiento, duración y repetición de los movimientos, cualidades motrices fundamentales, características de la frecuencia cardiaca, sistema energético predominante, tendencias actuales del alto rendimiento facilita, además de la caracterización exacta del deporte, la elaboración cuidadosa de los planes de entrenamiento (grupal e individual) y la determinación de los requisitos imprescindibles para los que se deben seleccionar y formar a los futuros atletas. Barbero, J., Barbero, M., Gómez y Castagna (2009) exponen que existe la necesidad de cuantificar el entrenamiento para programar las tareas y cargas externas que permitan una preparación óptima del deportista para la competición.

Se puede evidenciar en los conceptos anteriores que la caracterización del accionar competitivo es esencial para la actividad de los entrenadores y deportistas en el propósito de obtener altos resultados deportivos. En el tenis existe una considerable variación en el número de puntos disputados por los jugadores durante un partido, como mencionan Manno, R., Manno, V., Constantini, A. y Monferrer, M (1994) en el tenis se realizan carreras con cambios de dirección máxima velocidad y posteriormente un tiempo de recuperación.

Un ejemplo de un plan de entrenamiento bajo la aplicación del concepto de densidad lo menciona García Manso, J. (1996), definida como el aspecto cuantitativo del estímulo utilizado en el proceso de entrenamiento. Es empleada en una sesión de tenis en la que se va a entrenar el golpe de derecha cruzada y el criterio es tiempo; entonces se determinan cuatro series de tres minutos de repeticiones por dos minutos de pausa, esto sería igual a $(4 \times 3=12+8=20$ minutos de trabajo) de derecha cruzada.

Por su parte, Nacleiro, F. (2007) menciona que la densidad hace referencia a la relación entre la duración del esfuerzo y la duración de la pausa de recu- peración o descanso. Esta puede ser considerada densidad intra-sesión (relación pausa/esfuerzo inter-repeticiones, inter-series, inter-bloques o inter-ejercicios). Normalmente se expresa la duración de la misma en la micro-pausa (tiempo de recuperación entre series) y la macro-pausa (tiempo de recuperación entre ejercicios). Igualmente, la densidad también puede ser considerada como densidad inter-sesión (tiempo de recuperación entre sesiones o unidades de entrenamiento). Esta última está relacionada con la organización de la carga de entrenamiento (distribución e interconexión de las cargas en el tiempo) (Heredia, J. et ál. 2012).

La densidad (relación entre el esfuerzo y el descanso) y la frecuencia se relacionan de forma directamente proporcional. Por ello, a menor densidad (mucho descanso) menor frecuencia; a mayor densidad (poco descanso) mayor frecuencia.

En la siguiente tabla se exponen los comportamientos metabólicos respecto a la densidad del trabajo a realizar. El primer valor equivale a la proporción del tiempo de trabajo a alta intensidad y posterior el valor relacionado con el descanso.

Tabla 1. Relación del comportamiento metabólico frente a la densidad del trabajo.

\begin{tabular}{|c|c|}
\hline $\begin{array}{c}\text { OBJETIVOS / } \\
\text { METABOLISMOS }\end{array}$ & DENSIDAD \\
\hline Anaeróbico Aláctico & $1 / 4$ a $1 / 25$ \\
\hline Anaeróbico Láctico & $1 / 3$ a $1 / 10$ \\
\hline $\begin{array}{c}\text { Potencia Aeróbica } \\
\text { VO2 }\end{array}$ & $1 / 0,25$ a $1 / 2$ \\
\hline Mixto (aeróbico-anaeróbico) & $1 / 0$ (trabajo continuo) a $1 / 1$ \\
\hline Capacidad Aeróbica
\end{tabular}

Fuente: Matas, T. (2016)

Como se puede apreciar, la intensidad se relaciona con la densidad de entrenamiento. Debe haber más descanso cuanta más alta es la intensidad y esta se relaciona con la siguiente formula: Densidad= trabajo/ descanso. La densidad relativa da el porcentaje de la sesión en el que se está trabajando. Es el tiempo de trabajo respecto al trabajo total de una sesión.

De este modo, la variación de esta relación, al alargar o acortar el tiempo de la pausa de recuperación respecto a la duración del esfuerzo afectará a las res- 
puestas agudas (metabólicas, hormonales, cardiovasculares) y las adaptaciones provocadas por los estímulos de entrenamiento. Por tanto, dicha relación (estímulo/pausa) será dependiente del objetivo fisiológico y nivel de entrenamiento del sujeto.

El presente informe se realiza con el fin de caracterizar la densidad de las acciones competitivas de los jugadores de Croacia y Colombia en el contexto del primer día de enfrentamiento de la copa Davis 2017, realizada en Bogotá el 15 de septiembre. Los datos recopilados corresponden a los partidos efectuados entre los jugadores Alejandro González y Marin Čilić, y Santiago Giraldo y Franko Škugor en la modalidad de individuales.

La copa Davis es el evento de mayor trascendencia realizado en el tenis a nivel de naciones. De acuerdo con lo publicado en el sitio web Davis Cup (2017), la idea de una copa mundial de naciones se había originado antes por cuatro estudiantes de Harvard; uno de ellos, Dwight Davis, fue quien diseño el primer formato de competencia. Inicialmente el torneo se llamó el Lawn Tenis Challenge, pero por el trofeo aportado por Dwight Davis, modifico su nombre.

La forma en que funciona el ranking de la copa Davis refleja los resultados de las naciones participantes en los últimos cuatro años, pero tienen preponderancia los resultados más recientes. Se actualiza luego de cada eliminatoria, los resultados obtenidos van perdiendo valor a medida que pasa cada año, por esto cuentan más los puntos ganados en el año en curso.

Gracias al conocimiento de la importancia que tiene la caracterización de la actividad competitiva y el evento de copa Davis se realizó esta observación. A continuación, se expone la manera en que se efectuó y los resultados obtenidos.

\section{METODOLOGÍA}

Se utilizó como método la observación cuantitativa, que consiste en el registro sistemático, valido y confiable de comportamientos o conductas que se manifiestan (Hernández, Fernández y Baptista, 2006). En este caso, las conductas a observar fueron: la cantidad de puntos jugados, el marcador, el tipo de punto, dependiendo del número de golpes, siendo un punto corto de uno a tres golpes, punto medio de cuatro a seis golpes y punto largo más de seis golpes, la dura- ción de cada punto fue registrada por medio de un cronómetro. Todos los datos se registraron en una plantilla física para su recolección, posteriormente fueron tabulados en una matriz de Excel para su análisis e interpretación. Para la aplicación de la observación como método, se siguieron los pasos planteados por Estévez, M. (2003), para la aplicación de este método, que consisten en organización preliminar, ejecución de la observación, procesamiento de la información.

En la organización preliminar se determinó observar la densidad. Como medio se seleccionó la duración de los puntos, medidos con ayuda del cronómetro; se elaboró previamente la guía de observación; se entrenó a los observadores para la toma de los datos; se realizó un pilotaje previo de los instrumentos a emplear, los observadores y las condiciones de realización. La ejecución de la observación se realizó en el tiempo planificado y el escenario donde se desarrollaron los partidos (plaza de toros la Santa María de Bogotá).

El procesamiento de los resultados se realizó mediante el empleo de Microsoft Excel. Gracias a esta herramienta se hallaron datos de la estadística descriptiva: máximo, mínimo, promedio, desviación estándar, se realizó la distribución en puntos cortos, medios y largos, y se graficaron los resultados para facilitar su interpretación.

Esta observación estadística se realizó en tiempo real de juego, se utilizaron dos cronómetros. El primero, se inició cuando comenzó el partido y se detuvo cuando finalizó (para hallar el tiempo total). El segundo, se puso en marcha al iniciar cada punto y se detuvo cada vez que terminó; el tiempo total del game se tomó a partir de la sumatoria de tiempo de los puntos (permitió medir el tiempo real). Se registraron los puntos cortos (C), medios (M) y largos (L), para indicar la predominancia de cada uno de los jugadores.

\section{RESULTADOS DE LA OBSERVACIÓN}

Jugadores: Alejandro González (COL) contra Marin Čilić (HRV)

Fueron disputados dos partidos en esta primera jornada, el primero entre los tenistas Alejandro González (COL) y Marin Čilić (HRV), y el segundo entre Santiago Giraldo (Col) y Franko Škugor (HRV). 
El primer encuentro finalizó a favor del croata Čilić con marcador de 3 - 0 en sets y parciales de $6-1,6-4$ y 6 - 1. El partido se definió en sets consecutivos a favor de Croacia y por ende el primer punto de la confrontación. En el segundo encuentro de la jornada se jugó hasta un quinto set con marcador de 3 a 2 a favor del colombiano Santiago Giraldo y parciales de 6 - 4; 4-6; 5 - 7; 6 - 4 y $6-2$. En la tabla 2 se relaciona la cantidad total de puntos disputados para los encuentros y la cantidad de cada tipo de punto.

Tabla 2. Total y tipos de puntos disputados en los dos encuentros.

\begin{tabular}{|c|c|c|c|c|}
\hline \multirow{2}{*}{ Tipo de punto } & \multicolumn{2}{|c|}{ Partido 1} & Cantidad de puntos & Partido 2 \\
\cline { 2 - 5 } & Cantidad de puntos & $\%$ & 166 & $53.4 \%$ \\
\hline Corto & 66 & $21.1 \%$ & 98 & $31.5 \%$ \\
\hline Medio & 31 & $34 \%$ & 47 & $15.1 \%$ \\
\hline Largo & 50 & & 311 & \\
\hline Total & 147 & & & \\
\hline
\end{tabular}

Fuente: Elaboración propia.

El total de puntos jugados (147) en el primer encuentro en promedio tuvieron una duración de 6,7 $\pm 5,0$ segundos; el tiempo total del partido que fue de $1 \mathrm{~h} 52$ min. Se puede evidenciar que predominan los puntos cortos (66) que se aproximan al $50 \%$ del total de puntos jugados; seguidamente los puntos largos predominan con un $34 \%$ y menor presencia de los puntos medios con un $21.1 \%$. La duración de los puntos determina el tiempo real de juego que fue de $16 \mathrm{~min}$ 28 s 8 cs, lo que corresponde al 14,8 \% del tiempo total del partido.

Para el segundo encuentro fueron disputados 311 puntos en total que en promedio alcanzaban un tiempo de 5,5 $\pm 4,4$ segundos, en los cuales el predominante fue el tipo de punto corto con un total de 166 puntos, que corresponde al $53.4 \%$ del total sobre un $31.5 \%$ y un $15.1 \%$ para los puntos medios y largos respectivamente. Para este encuentro el tiempo total fue de $3 \mathrm{~h} 49 \mathrm{~min}$, mientras que el tiempo real fue de 28 min 29 s 8 cs, que equivale al 12,4 \% del tiempo total del partido (Figura 1.); a pesar de que la duración de ambos encuentros fue diferente, en términos de porcentaje, el tiempo real de juego fue muy similar para ambos casos.

En la tabla 3, se encuentran registrados la duración de los puntos, games y sets más largos y más cortos en los dos encuentros, principalmente se observa que para el primer encuentro el punto número 136 fue el más largo y los puntos 138 y 139 fueron los más cortos, del total de 147 puntos disputados; para el

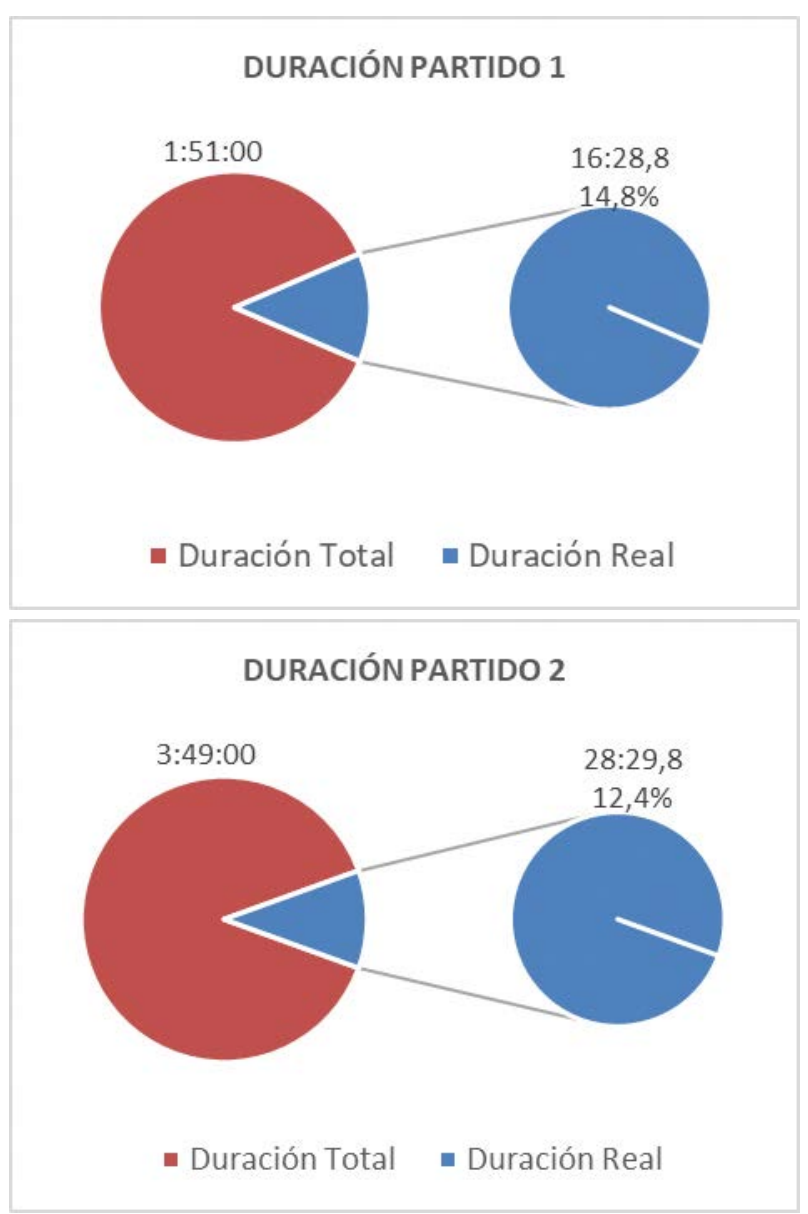

Figura 1. Relación entre el tiempo total y el tiempo real de juego de los dos partidos.

Fuente: Elaboración propia. 
segundo encuentro, el punto número 66 fue el más largo y el numero 194 el más corto del total de 311 puntos jugados. En ambos casos se puede evidenciar que los puntos de mayor duración están por debajo de los treinta segundos y que los puntos de menor duración están por debajo de un segundo.

Por su parte, los games más largos y más cortos se ubicaron hacia el final de los últimos sets, lo que podría indicar un nivel elevado de fatiga en estas instancias de manera que los puntos son definidos después de una larga consecución de golpes o, por el contrario, por un servicio efectivo.
Finalmente, para el caso de ambos encuentros, el tercer set fue el que tuvo la mayor duración, siendo muy similares los tiempos de un partido y otro. Para el caso de los sets más cortos o rápidos, estos fueron los disputados al inicio de los partidos, set uno y set dos para el primer encuentro y para el segundo encuentro respectivamente. Estos resultados pueden evidenciar una relación directamente proporcional entre el tiempo de duración de un set y el nivel de fatiga, a mayor fatiga mayor es el tiempo de duración del set mientras que a menor fatiga el set se desarrollara en menor tiempo.

Tabla 3. Duración de los puntos, games y sets jugados.

\begin{tabular}{|c|c|c|c|c|}
\hline \multirow{2}{*}{ Tipo de punto } & \multicolumn{2}{|c|}{ Partido 1} & \multicolumn{2}{|c|}{ Partido 2} \\
\hline & Duración & Ubicación ${ }^{7}$ & Duración & Ubicación \\
\hline Punto más largo (s) & $00: 25,5$ & \# 136 & $00: 22,8$ & \# 66 \\
\hline Punto más corto (s) & $00: 00,9$ & \# 138 у \# 139 & $00: 00,6$ & \# 194 \\
\hline Game más largo (min) & $02: 35,0$ & $5^{\circ}$ game $3^{\mathrm{er}}$ set & $02: 17,3$ & $6^{\circ}$ game del $5^{\circ}$ set \\
\hline Game más corto (s) & $00: 14,2$ & $6^{\circ}$ game $3^{\mathrm{er}}$ set & $00: 08,1$ & $5^{\circ}$ game del $4^{\circ}$ set \\
\hline Set más largo (min) & $06: 11,7$ & $3^{\mathrm{er}}$ set & $06: 10,9$ & $3^{\text {er }}$ set \\
\hline Set más corto (min) & $05: 04,2$ & $1^{\mathrm{er}} \operatorname{set}$ & $04: 41,4$ & $2^{\circ}$ set \\
\hline
\end{tabular}

Fuente: Elaboración propia.

En la comparación de los tiempos totales con los tiempos reales de cada set estos corresponden al $17 \%$, 13 \% y $15 \%$ respectivamente del tiempo total de cada set. En este partido en promedio la duración de un set fue de 05 min 29 s; siendo el tercero el único jugado por encima del promedio evidenciado en el gráfico 2.

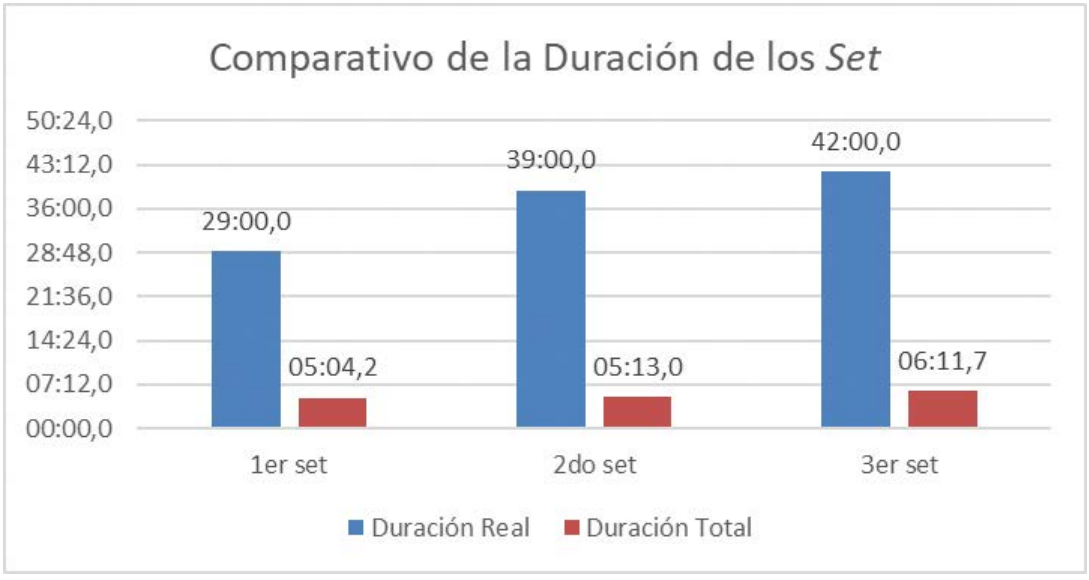

Figura 2. Comparación del tiempo total y real en cada set

Fuente: Elaboración propia.

7 Hace referencia al punto, game o set donde se dio el mayor o menor dato de la variable. 
En las tablas 4 y 5 se relacionan la cantidad y duración de los games para los sets disputados en ambos partidos. Para el caso del primer encuentro, el promedio de duración de cada game fue de 41 s 2 cs; 8 de los 24 games jugados estuvieron por arriba del promedio mientras que 16 se jugaros por debajo de este (tabla 4).

Para el caso del segundo encuentro, el promedio de duración de los games fue de 34 s 20 cs; del total de games disputados, 29 estuvieron por debajo de este promedio mientras que los 21 restantes se jugaron por encima del tiempo promedio (tabla 5).

Tabla 4. Duración (s) de los games en cada set del primer partido.

\begin{tabular}{|c|c|c|c|}
\hline Game \# & $\mathbf{1}^{\text {er }}$ set & $\mathbf{2}^{\mathbf{o}}$ set & $\mathbf{3}^{\text {er }}$ set $^{{ }^{\prime}}$ \\
\hline 1 & $00: 46,4$ & $01: 13,6$ & $00: 36,2$ \\
\hline 2 & $00: 51,2$ & $00: 17,3$ & $00: 46,7$ \\
\hline 3 & $00: 18,0$ & $00: 29,7$ & $00: 24,0$ \\
\hline 4 & $00: 52,8$ & $00: 29,7$ & $00: 58,4$ \\
\hline 5 & $00: 20,5$ & $00: 30,1$ & $02: 35,0$ \\
\hline 6 & $01: 19,3$ & $00: 19,4$ & $00: 14,2$ \\
\hline 7 & $00: 36,0$ & $00: 19,7$ & $00: 37,3$ \\
\hline 8 & & $00: 27,1$ & \\
\hline 9 & & $00: 40,8$ & \\
\hline 10 & & $00: 25,6$ & \\
\hline
\end{tabular}

Fuente: Elaboración propia.

Tabla 5. Duración (s) de los games en cada set del segundo partido.

\begin{tabular}{|l|l|l|l|l|l|}
\hline \multicolumn{1}{|c|}{ Game \# } & \multicolumn{1}{c|}{$\mathbf{1}^{\text {er }}$ set } & \multicolumn{1}{c|}{$\mathbf{2}^{\text {o } \text { set }}$} & \multicolumn{1}{c|}{$\mathbf{3}^{\text {er }}$ set } & \multicolumn{1}{|c|}{$\mathbf{4}^{\text {o }}$ set $^{\text {set }}$} \\
\hline 1 & $00: 44,3$ & $00: 20,6$ & $00: 45,8$ & $00: 45,4$ & $00: 40,0$ \\
\hline 2 & $00: 23,1$ & $00: 40,3$ & $00: 23,7$ & $00: 59,1$ & $00: 25,7$ \\
\hline 3 & $00: 46,3$ & $00: 11,6$ & $00: 23,5$ & $00: 29,6$ & $00: 19,5$ \\
\hline 4 & $01: 02,5$ & $00: 15,3$ & $00: 29,5$ & $00: 51,4$ & $00: 23,9$ \\
\hline 5 & $00: 15,1$ & $00: 27,3$ & $00: 19,2$ & $00: 08,1$ & $00: 21,0$ \\
\hline 6 & $00: 23,1$ & $00: 36,2$ & $00: 44,5$ & $00: 47,5$ & $02: 17,3$ \\
\hline 7 & $00: 20,1$ & $00: 27,2$ & $00: 17,1$ & $00: 21,0$ & $00: 10,1$ \\
\hline 8 & $00: 38,4$ & $00: 47,0$ & $01: 00,6$ & $00: 37,1$ & $00: 54,4$ \\
\hline 9 & $00: 44,4$ & $00: 24,5$ & $00: 13,6$ & $00: 27,2$ & \\
\hline 10 & $00: 50,0$ & $00: 31,4$ & $00: 29,0$ & $00: 32,0$ & \\
\hline 11 & & & $00: 08,5$ & & \\
\hline 12 & & & $00: 56,1$ & & \\
\hline
\end{tabular}

Fuente: Elaboración propia.

La densidad competitiva del primer partido se dio de la siguiente manera: el total de puntos fue 147, con un marcador de 3 sets a 0 a favor de Croacia con parciales de $6-1,6-4,6-1$; el tiempo total de juego fue de $1 \mathrm{~h}$ 
51 min $00 \mathrm{~s}$, mientras que el tiempo real de juego fue 16 min 28 s 8 cs, distribuido en un primer set de 05 min 04 s 2 cs, un segundo de 05 min 13 s 0 cs y un tercero de 06 min $11 \mathrm{~s} 7$ cs. De esta manera, $02 \mathrm{~min}$ 35 s 0 cs fue la duración del game más largo y, por tanto, 14 s 2 cs el lapso del game más corto.

En cuanto a la densidad competitiva del segundo partido, fue un total de 311 puntos jugados hasta un quinto set con marcador de 3 a 2 a favor del Colombiano Giraldo con parciales de 6 - 4, 4-6, 5-7,6-4 y 6-2; el tiempo total de juego fue de $3 \mathrm{~h} 00 \mathrm{~min} 49$ $\mathrm{s}$, mientras que el tiempo real de juego fue $28 \mathrm{~min} 29$ s, distribuido en un primer set de 06 min 07 s 3 cs, un segundo de $04 \mathrm{~min} 41 \mathrm{~s} 4 \mathrm{cs}$, un tercero de $06 \mathrm{~min} 10$ s $9 \mathrm{cs}$, un cuarto set de 05 min 58 s 02 cs, y un último y quinto set de 05 min $32 \mathrm{~s}$. De esta manera, el quinto game del cuarto set con 08 seg 1 cs fue el más corto $\mathrm{y}$, por tanto, el sexto game del quinto set con $02 \mathrm{~min}$ 17 s 3 cs, el más largo.

\section{DISCUSIÓN}

La superficie de juego tiene un papel importante y afecta de cierta manera el desarrollo de un encuentro de tenis, puntos más largos y con mayor número de golpes suceden en superficies lentas (polvo de ladrillo) como la utilizada en los encuentros entre Colombia y Croacia, en comparación a otras superficies donde el juego es más veloz (Fernández-Fernández, Sanz-Rivas y Méndez-Villanueva, 2009).

Para Smekal et ál (2001) a partir de su estudio desarrollado con 20 jugadores de tenis en campo de superficie de polvo de ladrillo y teniendo en cuenta las características de juego de los deportistas (defensivos u ofensivos) determinaron un tiempo de duración de los puntos de 8,2 $\pm 5,1$ segundos para los defensivos y 4,8 $\pm 1,8$ segundos para los ofensivos. También, el porcentaje correspondiente al tiempo efectivo de juego fue de 29,3 $\pm 12,1 \%$ para los jugadores defensivos y de 20,3 $\pm 8,2$ para los jugadores ofensivos.

En otros estudios, fueron encontrados valores promedio de duración de un punto en $7,5 \pm 7,3$ segundos, un porcentaje de tiempo efectivo de juego de 18,2 \pm 5,8 \% y una densidad de 1:2.2 (Fernández, J., Fernández-García, B., Méndez-Villanueva, A. y Terrados, N. 2005). Además, en el estudio realizado por
Méndez-Villanueva, A., Fernández-Fernández, J. y Bishop, D. (2007) el tiempo efectivo de juego correspondió a un 21,5\% del total, una densidad 1:2.2 y un tiempo promedio por punto de 7,5 segundos. También, Fernández-Fernández et ál. (2008) halló un perfil de actividad con una densidad de 1:2.1, un tiempo efectivo de juego de $21,6 \pm 6,1 \%$ y un promedio de tiempo de 7,2 $\pm 5,2$ segundos por punto.

Finalmente, otros autores se han centrado únicamente en determinar la duración promedio de un punto o rally para el caso de superficies de juego lentas como lo es la de polvo de ladrillo; O’Donoghue y Ingram (2001) establecieron la duración promedio en 7,7 $\pm 1,7$ segundos; Girard y Miller (2004) mencionan un tiempo promedio de 7,2 \pm 1,7 segundos, Hornery, D. J., Farrow, D., Mujika, I. y Young, W. (2007) establecieron como promedio de duración 7,5 segundos y, por último, Murias, J. M., Lanatta, D., Arcuri, C. R. y Laino, F. A. (2007) promediaron un tiempo de 7,4 segundos.

A partir de las relaciones establecidas con los estudios revisados, el tiempo promedio de los puntos fue un poco menor, pero no tan lejano a los mencionados anteriormente, el valor más cercano fue el encontrado en el estudio de Smekal et ál. (2001) para los tenistas ofensivos. En cuanto al porcentaje real o efectivo de juego para los dos partidos de Copa Davis 2017 analizados fue menor en comparación a los valores de referencia de los estudios consultados; por último, en comparación a la densidad establecida en los estudios de referencia y la densidad de los encuentros disputados entre Colombia y Croacia podría decirse que fueron disputados con una menor intensidad.

\section{CONCLUSIONES}

Los resultados logrados en el presente estudio demuestran que el perfil de actividad en el tenis es de naturaleza intermitente, con una demanda esfuerzo-descanso de 1:5 para el primer partido y 1:7 para el segundo partido. Este ejercicio intermitente según Argemi, R. (2010), implica momentos cortos de esfuerzo con o sin elemento y tiene características que se diferencian en los sistemas energéticos.

Los puntos cortos predominaron en los dos partidos, por lo que el resultado de los encuentros fue dado 
por las condiciones físicas de orden explosivo de los tenistas y por su eficiencia en la precisión de ubicación de la bola y en la rápida definición de los puntos. De esta manera, en el primer partido el Croata tuvo la ventaja desde el principio dadas sus fortalezas físicas y su estilo de juego lanzado al ataque y a la rápida definición de cada punto; por el contrario, la táctica de juego Colombiano se enfocaba en la defensa y en mantener la bola en juego el mayor tiempo posible, lo cual no iba acorde con el tipo de rival.

En el segundo partido se planteó una estrategia más acorde al rival por parte del equipo colombiano. El tenista local planteó un partido donde él tomó la iniciativa (por lo menos en los primeros dos sets) y no permitió que su rival sacara provecho de sus condiciones físicas, entendió que los puntos había que definirlos rápidamente y se valió de la buena precisión de sus golpes para sacar ventaja.

El tenis es un deporte que demanda una condición física excepcional a sus practicantes. Los resultados de la medición de la densidad competitiva corroboran que el tenis está basado en esfuerzos de tipo explosivo, basado en sistema anaeróbico alactácido, esfuerzos de $11 \mathrm{~s}-50 \mathrm{~s}$ (tiempo en actividad por set), por la vía metabólica de los fosfórenos. Sin embargo, es muy dependiente de una base aeróbica de alta capacidad que permite recuperarse lo más rápidamente posible en cada break y al finalizar el partido pensando en el próximo encuentro que, generalmente, es al día siguiente.

\section{REFERENCIAS}

Argemi, R. (2010). Ejercicio intermitente en deportes de conjunto. Análisis y aplicación en el proceso de entrenamiento deportivo. http://www.deportesaciclicos.com/ uploadfiles/argemiresumen.pdf

Barbero, J., Barbero, V., Gómez, M. y Castagna, C. (2009). Análisis cinemático del perfil de actividad en jugadoras infantiles de fútbol mediante tecnología GPS. Kronos: La Revista Científica de Actividad Física y Deporte, 15, 35-42.

Buitrago, J. (2016). Programa de formación deportiva del tenista para las categorías de base en la fundación transformando vidas de Boyacá, Colombia. [Tesis de doctorado, Universidad de Ciencias de la Cultura Física y el Deporte "Manuel Fajardo"].
Davis Cup. (2017). Historia de la copa Davis. https:// www.daviscup.com/es/organizaci\%C3\%B3n/historia-de-la-copa-davis.aspx

Estévez, M. (2003). Metodología de la investigación. Recopilación temática del programa docente de la especialidad de medicina del deporte. La Habana: Facultad de Ciencia Médicas Enrique Cabrera.

Fernández, J., Fernández-García, B., Méndez-Villanueva, A. y Terrados, N. (2005). Activity patterns, lactate profiles and ratings of perceived exertion (RPE) during a professional tennis singles tournament. In Quality coaching for the future. 14th ITF worldwide coaches workshop. London: ITF.

Fernández-Fernández, J., Sanz-Rivas, D., Fernández-García, B. y Méndez-Villanueva, A. (2008). Match activity and physiological load during a clay- court tennis tournament in elite female players. Journal of Sports Sciences, 26(14), 1589-1595. https://doi. org/10.1080/02640410802287089

Fernández-Fernández, J., Sanz-Rivas, D. y Méndez-Villanueva, A. (2009). A Review of the Activity Profile and Physiological Demands of Tennis Match Play. Strength and Conditioning Journal, 31(4), 15-26.

García Manso, J., Navarro Valdivieso, M. y Ruiz Caballero, J. (1996). Planificación del entrenamiento deportivo. Madrid, España: Gymnos Editorial.

Girard, O. y Millet, G. P. (2004, October). Effects of the ground surface on the physiological siological and technical responses in young tennis players. In Science and Racket Sports III: The Proceedings of the Eighth International Table Tennis Federation Sports Science Congress and The Third World Congress of Science and Racket Sports (p. 49). Routledge.

Hernández Sampieri, R., Fernández Collado, C. y Baptista Lucio, P. (2006). Metodología de la investigación. México: Editorial Mc Graw Hill.

Hornery, D. J., Farrow, D., Mujika, I. y Young, W. (2007). An integrated physiological and performance profile of professional tennis. British journal of sports medicine, 41(8), 531-536.

Manno, R., Manno, V., Constantini, A. y Monferrer, M. (1994). Fundamentos del entrenamiento deportivo. Barcelona, España: Paidotribo.

Matas Barcelo, T. (2016). Teoría entrenamiento y preparación física. Tema 5: Carga entrenamiento y sus componentes. http://www.tonimatasbarcelo.com/teoria-entrenamiento-y-preparacion-fisica-tema-5-carga-entrenamiento-y-componentes/ 
Méndez-Villanueva, A., Fernández-Fernández, J. y Bishop, D. (2007). Exercise-induced homeostatic perturbations provoked by singles tennis match play with reference to development of fatigue. British journal of sports medicine, 41(11), 717-722.

Murias, J. M., Lanatta, D., Arcuri, C. R. y Laino, F. A. (2007). Metabolic and functional responses playing tennis on different surfaces. Journal of strength and conditioning research, 21(1), 112.

Nacleiro Ayllón, F. (2007). Entrenamiento de fuerza y prescripción del ejercicio. En: Jiménez Gutiérrez, A. Entrenamiento personal. Bases, fundamentos y aplicaciones (pp. 87-134). Barcelona: INDE Publicaciones.

O’Donoghue, P. y Ingram, B. (2001). A notational analysis of elite tennis strategy. Journal of sports sciences, 19(2), 107-115.
Heredia, J., Isidro, F., Peña, G., Mata, F., Moral, S., Martín, M., Segarra, V. y Da Silva, M. (2012). Criterios básicos para el diseño de programas de acondicionamiento neuromuscular saludable en centros de fitness. EFDeportes. com, Revista digital, 170 (17). https://www.efdeportes.com/efd170/diseno-de-programas-de-acondicionamiento-neuromuscular.htm

Smekal, G., Von Duvillard, S., Rihacek, C., Pokan, R., Hofmann, P., Baron, R., ... Bachl, N. (2001). A physiological profile of tennis match play. Physical Fitness and Performance, 33(6), 999-1005.

Zatsiorsky, V. (1989). Metrología deportiva. Moscú: Editorial Planeta. 